\title{
Deriving Hurford's Constraint *
}

\author{
Marie-Christine Meyer \\ The Hebrew University of Jerusalem
}

\begin{abstract}
I show that the infelicity of disjunctions in which one disjunct entails the other ("Hurford disjunctions"), as well as the felicity of a subclass of Hurford disjunctions (e.g., some or all), can be derived from a general principle of Brevity under the independently motivated assumption that uncertainty implicatures are generated in the grammar.
\end{abstract}

Keywords: implicatures, Hurford's constraint, disjunction, exhaustification, maxim of brevity, ignorance implicatures

\section{Introduction}

I want to begin by comparing two minimally different assertions:

(1) a. Mary drank some of the beers.

b. Mary drank some or all of the beers.

The Venn-diagram in Figure 1 depicts the set of situations in which the two sentences are true (I will use SOME/ALL as transparent abbreviations for whole sentences).

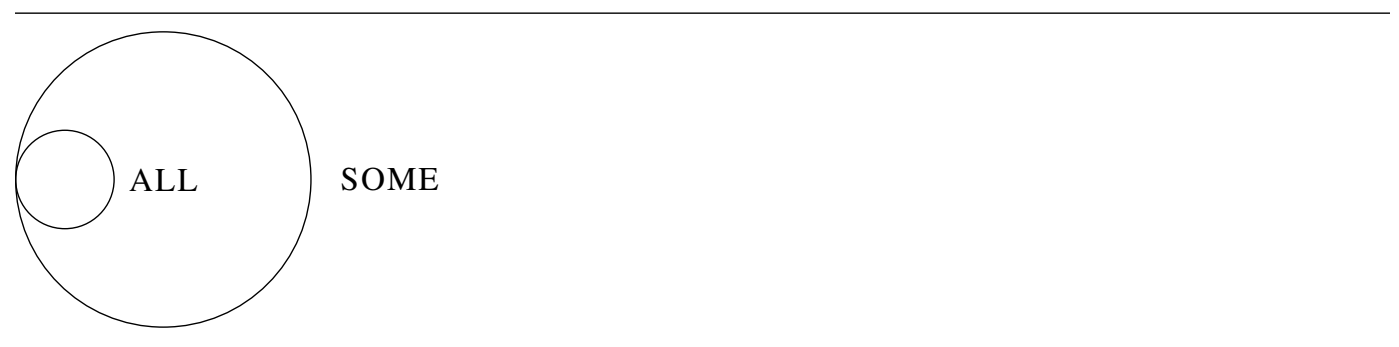

Figure 1 Semantic relation between propositions in (1)

As the diagram illustrates, (1a) and (1b) are semantically equivalent under any standard semantics for or (e.g., logical $\vee$ ). Our locus of comparison, by contrast,

* For their helpful comments and criticism, I thank Irene Heim, Danny Fox, Uli Sauerland, Kai von Fintel, Luka Crnič, Orin Percus, Aynat Rubinstein, Philippe Schlenker, Lavi Wolf, and Seth Yalcin. I am also grateful to the audience and reviewers at SALT 24 for insightful questions and comments. 
will be the pragmatics. More specifically, it is the predictions of the two major schools of thought on implicature: the Neo-Gricean theory on the one hand, and the grammatical theory of scalar implicatures on the other hand.

Consider the predictions of the Neo-Gricean theory first. Under this theory, implicatures arise after semantic computation, through competition of what has been asserted to what could have been asserted, given language-independent constraints. To be precise:

Neo-Gricean Cooperative Principle

The speaker should assert $\alpha$ instead of $\phi$ if:

a. $\alpha$ is relevant in $C$

b. $\llbracket \alpha \rrbracket \nsubseteq \llbracket \phi \rrbracket$

c. $\alpha \in \mathcal{A L T}(\phi)$

d. the speaker is certain that $\alpha$ (which we will abbreviate as $\mathrm{K}(\alpha)$ )

Following Katzir (2007), I take (2c) to be definable in terms of syntactic complexity, as spelled out below (simplifying Katzir's proposal slightly):

(3) Formal Alternatives $\mathcal{A} L T$

(Katzir 2007)

If a structure $\alpha$ can be derived from $\phi$ by substituting terminal nodes in $\phi$ with lexical items or with subconstituents of $\phi$, or by deleting subconstituents of $\phi$, then $\alpha \in \mathcal{A L T}(\phi)$

Let us quickly review the well-known predictions that emerge for the original simple sentence SOME in (1a). The condition in (2c) rules out competition of this sentence with the alternative in (4b), while allowing competition with the universal alternative ALL in (4a): ${ }^{1}$

1 The question as to how pragmatic competition can be restricted in this way is known as the Symmetry Problem. The Neo-Gricean solution has the form of an ad absurdum argument: suppose there was no restriction that $\alpha \in \mathcal{A} L T(\phi)$ as defined by (3). Then (1) would compete with both alternatives (0a) and $(0 b)$ - both are equally relevant and more informative:

(i) Mary drank some of the beers.

a. Mary drank all of the beers.

b. Mary drank some but not all of the beers.

Via the Cooperative Principle, the following uncertainty implicatures would be predicted:

(ii) a. $\neg \mathrm{K}$ (Mary drank all of the beers)

b. $\neg \mathrm{K}$ (Mary drank some but not all of the beers)

As we will see shortly, however, these two uncertainty implicatures make it impossible to derive a scalar implicature of the form $\mathrm{K} \neg$ (ALL). But (1) does give rise to this scalar implicature. Therefore, so the argument, these cannot both be competing alternatives. 
Deriving Hurford's Constraint

(4) Mary drank some of the beers.

a. Mary drank all of the beers. $\checkmark$

b. Mary drank some but not all of the beers. $\boldsymbol{X}$

Given that (4a) is not only part of $\mathcal{A} L T$ but also meets the other conditions in (2), the only reason a cooperative speaker would not assert it must be the last condition of the Cooperative Principle $((2 \mathrm{~d}))$. The result of this reasoning is an implicature:

Mary drank some of the beers.

$\leadsto \neg \mathrm{K}$ (Mary drank all of the beers)

The speaker is not certain that Mary drank all of the beers

$\mathrm{K}$ is a universal operator quantifying over the speaker's doxastic alternatives. At this point, it will be useful to introduce the following terminology:

(6) Inventory of Implicatures
a. Uncertainty: $\neg \mathrm{K} \alpha$
b. Ignorance: $\neg \mathrm{K} \alpha \wedge \neg \mathrm{K} \neg \alpha$
c. Scalar: $\mathrm{K} \neg \alpha$

Thus, an uncertainty implicature $\neg \mathrm{K} \alpha$ makes a weak statement in that it is consistent with two kinds of situations: those in which the speaker is certain that $\alpha$ is false (= $\mathrm{K} \neg \alpha$ ), and those situations in which the speaker's alternatives contain both $\alpha$ - and $\neg \alpha$-worlds $(=\neg \mathrm{K} \alpha \wedge \neg \mathrm{K}(\neg \alpha))$.

Is the uncertainty implicature in (5) empirically adequate? Perhaps. More importantly at this point, the scalar implicature which the sentence undoubtedly can have is not yet derived. Next to the Cooperative Principle and the structural definition of $\mathcal{A} L T$, it is the third standard ingredient in the Neo-Gricean account which will derive this:

\section{Epistemic Step} (Sauerland 2004) Given the assertion of $\phi$ in context $\mathrm{C}$, and the associated uncertainty implicatures $\neg \mathrm{K} \alpha_{1}, \ldots, \neg \mathrm{K} \alpha_{n}, \neg \mathrm{K} \alpha_{k}$ can be strengthened into $\mathrm{K}\left(\neg \alpha_{k}\right)$ only if the result is consistent with the sum of all uncertainty implicatures of $\phi$ in $\mathrm{C}$ and $\mathrm{K} \phi$

The Epistemic Step, then, describes the third and final step in the pragmatic implicature computation. Whether the hearer takes this step is dependent on contextual considerations about the speaker's state of mind (see Soames 1982): 
Opinionated Speaker

A speaker is opinionated about $\alpha$ if it holds that $\mathrm{K} \alpha \vee \mathrm{K} \neg \alpha$

The uncertainty implicature $\neg \mathrm{K} \alpha$ combined with the assumption that the speaker is opinionated logically entails the scalar implicature $\mathrm{K} \neg \alpha$. In other words, a hearer who has derived an uncertainty implicature and furthermore assumes the speaker to be opinionated will take the Epistemic Step iff the consistency requirements given in (7) are met. Summing up, the simple sentence (1a) is predicted to have either a weak implicature $\neg \mathrm{KALL}-$ if the hearer does not assume the speaker to be opinionated — or else a scalar implicature $\mathrm{K} \neg(\mathrm{ALL})$.

Having the three essential building blocks of the theory in place - the Cooperative Principle, the definition of structural alternatives $\mathcal{A} L T$, and a strengthening mechanism like the Epistemic Step - we can check what this theory would predict for the more complex sentence SOME OR ALL in (1b). As illustrated in Figure 1, this sentence is semantically equivalent to the simpler version SOME. Therefore, it will have the same competing alternative as the simpler sentence, namely, ALL. This alternative fulfills all conditions of the cooperative principle, in particular, it is structurally less complex and thus part of $\mathcal{A} L T$ (SOME OR ALL). In the by now familiar fashion, we can derive the following uncertainty implicature:

Mary drank some or all of the beers.

$\leadsto \neg \mathrm{K}(\mathrm{ALL})$

Crucially, now, we can even take the Epistemic Step:

$$
\mathrm{K}(\mathrm{SOME} \text { or } \mathrm{ALL}) \wedge \neg \mathrm{K}(\mathrm{ALL}) \wedge \mathrm{K} \neg(\mathrm{ALL}) \checkmark
$$

There is simply nothing in the theory which would prevent this scalar implicature. Needless to say, this is a wrong result: the complex sentence SOME OR ALL can never give rise to this scalar implicature. In conclusion, then, the prediction of the Neo-Gricean theory regarding the difference between SOME and SOME OR ALL is that both are not only semantically equivalent, but may even give rise to the same (scalar) implicature, contrary to fact (this observation has also been made independently in Alonso-Ovalle (2006: 56ff.)).

Let us now turn to the predictions of the grammatical theory, of which I take Fox (2007) to be the standard version. Fox rejects the idea that pragmatic competition is subject to the brevity condition in (2c). The Cooperative Principle therefore becomes:

(11) Fox-Grice Cooperative Principle

The speaker should assert $\alpha$ instead of $\phi$ if:

a. $\alpha$ is relevant in $C$ 
Deriving Hurford's Constraint
b. $\llbracket \alpha \rrbracket \varsubsetneqq \llbracket \phi \rrbracket$
c. $\mathrm{K} \alpha$

Crucially, this version of the Cooperative Principle no longer allows the strengthening of the Epistemic Step, i.e., derivation of scalar implicatures from simple sentences like Mary drank some of the beers if we assume that all other building blocks of the Neo-Gricean theory remain intact. The problem is the symmetry of alternatives (see fn. 1). (11) predicts that SOME will compete will all relevant stronger alternatives, i.e. SOME BUT NOT ALL and ALL (see (4)). This will result in two kinds of uncertainty implicatures:

$$
\begin{aligned}
& \text { a. } \neg \mathrm{K} \text { (Mary drank all of the beers) } \\
& \text { b. } \neg \mathrm{K} \text { (Mary drank some but not all of the beers) }
\end{aligned}
$$

But these cannot be strengthened into scalar implicatures: if the speaker is certain of his original assertion (= KSOME), and if he were certain that Mary didn't drink all of the beers $(\mathrm{K} \neg(\mathrm{ALL})$ ), it would follow that he is certain that Mary drank some but not all of the beers (=KSOME BUT NOT ALL). But this contradicts the second uncertainty implicature $\neg$ KSOME BUT NOT ALL, and therefore violates the consistency condition on the Epistemic Step. What we are left with is a strengthening of a different kind: (13) and (12b) together with the assertion entail that the speaker is truly ignorant with respect to stronger alternatives:

$$
\neg \mathrm{K} \text { (Mary drank all of the beers) \& } \neg \mathrm{K} \neg \text { (Mary drank all of the beers) }
$$

Summarizing this intermediate result, under the view advocated in Fox (2007), the simple sentence SOME (see (1a)) may give rise to a true ignorance implicature as in (13). Pragmatic reasoning alone will never give rise to a scalar implicature. It is at this point that the theory becomes grammatical: scalar implicatures are in fact derived in the semantics, through a covert exhaustivity operator exh (see Chierchia (2006); Fox (2007)):

$$
\llbracket \operatorname{exh} \phi \rrbracket=\phi \& \forall \alpha \in I E\left(\phi, \mathcal{A} L T_{\phi}\right): \neg \alpha
$$

Importantly, the set of formal alternatives $\mathcal{A} L T$ and, consequently its subset $I E(\phi, \mathcal{A L T})$ of innocently excludable alternatives is defined exactly as in the Neo-Gricean theory, i.e. in terms of syntactic complexity. Simplifying, an alternative $\alpha$ is innocently excludable if it can be negated without logical contradiction:

$$
I E\left(\phi, \mathcal{A} L T_{\phi}\right)=\left\{\psi \mid \llbracket \phi \rrbracket \varsubsetneqq \llbracket \psi \rrbracket \wedge \neg \exists \psi^{\prime} \in \mathcal{A} L T(\phi) \text { s.t. }(\llbracket \phi \rrbracket \cap \llbracket \neg \psi \rrbracket) \subseteq \llbracket \psi^{\prime} \rrbracket\right\}
$$

Given the availability of the operator exh, the original simple sentence (1a) is structurally ambiguous between two different LFs, the semantics of which are given below: 

a. $\llbracket \operatorname{exh} \mathrm{SOME} \rrbracket=\mathrm{SOME} \wedge \neg \mathrm{ALL}$
b. $\llbracket \mathrm{SOME} \rrbracket=\mathrm{SOME}$

Suppose we chose the second LF in (16b). Once the sentence is sent off to the pragmatics, it will compete with the two stronger symmetric alternatives seen in (12) with the result reviewed above, namely, a true ignorance implicature:

(17) Mary drank some of the beers

$$
\leadsto \neg \mathrm{K}(\mathrm{ALL}) \wedge \neg \mathrm{K} \neg(\mathrm{ALL})
$$

For the LF in (16a), on the other hand, there is no equally relevant, stronger alternative, so that there will be no pragmatic implicatures for this LF.

Let us now check the predictions of the grammatical theory for the more complex sentence in(1b), repeated again below:

Mary drank some or all of the beers. (= SOME OR ALL)

Suppose we adjoin exh to this sentence. As illustrated below, this would result in the same false prediction as the pragmatic theory, namely, that the complex sentence could give rise to the scalar implicature that Mary did not drink all of the beers:

$$
\begin{aligned}
& \llbracket \text { exh } \text { SOME or ALL } \rrbracket= \\
& \mathcal{A} L T=\{\text { SOME, ALL }\} \\
& I E=\{\text { ALL }\} \\
& =\operatorname{SOME} \wedge \neg \text { ALL } \boldsymbol{x}
\end{aligned}
$$

As opposed to the pragmatic theory, however, the grammatical theory has a way to avoid this false prediction. The solution comes from a well-known constraint on disjunctions, first discussed and named after Hurford (1974):

\section{(20) Hurford's Constraint (HC)}

A disjunction in which one disjunct entails the other is ill-formed

Quite obviously, ALL entails SOME. Given the generalization in (20), we are thus left wondering why the corresponding sentences like our original (1b) are felicitous. The grammatical theory's answer to (19) likewise entails an answer to this puzzle: there is an LF which complies with (20):

$$
\text { [exh SOME] or [ALL] }
$$

In this structure, the exhaustivity operator is embedded inside the first disjunct. The first disjunct will therefore no longer entail ALL:

$$
\begin{aligned}
& \llbracket[\operatorname{exh} \mathrm{SOME}] \text { or }[\mathrm{ALL}] \rrbracket=(\mathrm{SOME} \wedge \neg \mathrm{ALL}) \vee \mathrm{ALL} \\
& \equiv \mathrm{SOME}
\end{aligned}
$$


Deriving Hurford's Constraint

Given the constraint in (20), the LF in (21) will be the only available structure for the complex sentence, yielding the semantics given above. What, then, are the predicted pragmatic implicatures for this structure? The set of relevant and stronger alternatives is given below — note the parallelism to the simple LF SOME in (16b):

$$
A L T_{P}=\{\text { SOME but not ALL, ALL }\}
$$

As is familiar by now, this set of alternatives will give rise to ignorance implicatures:

$$
\begin{aligned}
& {[\operatorname{exh} \mathrm{SOME}] \text { or } \mathrm{ALL}} \\
& \sim \neg \mathrm{K} \mathrm{ALL} \wedge \neg \mathrm{K} \neg \mathrm{ALL}
\end{aligned}
$$

These are exactly the same (pragmatic) implicatures as are predicted to arise for the simpler sentence SOME, as reviewed above. Thus, the grammatical theory predicts that both sentences may be completely equivalent both semantically and pragmatically. The predictions are summarized below:

\section{(25) Neo-Gricean Theory of Implicatures}

a. SOME

Uncertainty Implicature $\neg \mathrm{K}$ (ALL), Scalar implicature $\mathrm{K} \neg$ (ALL)

b. SOME or ALL

Uncertainty Implicature $\neg \mathrm{K}(\mathrm{ALL}), *$ Scalar implicature $\mathrm{K} \neg(\mathrm{ALL})$

\section{Grammatical cum pre-Neo-Gricean Theory of Implicatures}

a. SOME

Ignorance Implicature $\neg \mathrm{K}(\mathrm{ALL}) \wedge \neg \mathrm{K} \neg(\mathrm{ALL})$, Scalar implicature $\mathrm{K} \neg(\mathrm{ALL})$

b. SOME or ALL

Ignorance Implicature $\neg \mathrm{K}(\mathrm{ALL}) \wedge \neg \mathrm{K} \neg(\mathrm{ALL})$

\section{Grammatical uncertainty implicatures}

In this section I will introduce the basics of a new grammatical theory of implicature (see Meyer (2013)). The idea is that all implicatures - including uncertainty and ignorance implicatures - are derived through exhaustification in the grammar. First, we assume that all assertively used sentences are in fact covertly modalized by an underlying (possibly evidential) operator which I call the Matrix K operator:

\section{Matrix K Axiom}

Assertion of $\phi$ by $\mathrm{S}$ is parsed as $\mathrm{K}_{s} \phi$ at LF 
As before I will usually leave out the subscript $s$ since $\mathrm{K}$ is interpreted relative to the speaker by default. Again, just as before, $\mathrm{K}$ is a universal quantifier over belief-worlds.

When combined with the general availability of exhaustification at propositional nodes, the Matrix K Axiom allows for different scopal orderings between exh and $\mathrm{K}$ at the level of logical form. Independent (pragmatic) principles are expected to systematically restrict these possibilities. In the basic version of the theory outlined here, we have only one pragmatic principle:

\section{Epistemic Transparency (ET)}

An LF of the form $\left[\ldots \mathrm{K}_{x} \phi\right]$ is licensed iff it entails $x$ 's epistemic attitude about every $\psi \in \mathcal{A} L T(\phi)$

Before checking the predictions of this principle, it should be noted that structural alternatives $\mathcal{A} L T$ are defined as before, in terms of Katzir's notion of structural complexity (see (3) above). To this I add an additional restriction on the algorithm deriving $\mathcal{A} L T:^{2}$

\section{(29) Restriction on ALT}

DELETE \& REPLACE may not target the K operator

Let us now go through some examples on how to apply the structural definition of $\mathcal{A} L T$ in a system with Matrix $\mathrm{K}$ and (embedded) exh. When exh is adjoined only once, above $\mathrm{K}$, the formal alternatives it quantifies over are as expected:

$$
\begin{aligned}
& \operatorname{exh} \mathrm{K}[\mathrm{A} \text { or } \mathrm{B}] \\
& \mathcal{A} L T=\{\mathrm{K} \mathrm{A}, \mathrm{K} B, \mathrm{~K}[\mathrm{~A} \text { and } \mathrm{B}]\}
\end{aligned}
$$

When one exhaustivity operator is in the scope of another, there will in principle be more alternatives which exh could negate:

$$
\begin{aligned}
& \operatorname{exh}_{2} \mathrm{~K} \operatorname{exh}_{1}[\mathrm{~A} \text { or B] } \\
& \mathcal{A} L T_{1}=\left\{\mathrm{K} \operatorname{exh}_{1} \mathrm{~A}, \mathrm{~K} \operatorname{exh}_{1} \mathrm{~B}, \mathrm{~K} \operatorname{exh}_{1}[\mathrm{~A} \text { and B], K A, K B, .. }\}\right.
\end{aligned}
$$

The basics of the theory can now be applied to a few examples. Consider the following LFs:
a. $\mathrm{K}[\mathrm{A}$ or $\mathrm{B}]$
b. K SOME

2 Ultimately, this restriction should be derived from independent facts about the Matrix K operator, but here I will make do with the stipulation in (29). 
If these were licensed, a simple disjunction or a simple sentence sOME would be predicted to be felicitous if the speaker is certain that A AND B is true, or if she is certain that ALL. The principle of Epistemic Transparency correctly rules out these LFs. This is because they don't entail an epistemic attitude about the relevant alternatives, e.g., A AND B and ALL.

What are licensed LFs in the theory? We will start with a simple disjunction. There are exactly two kinds of LFs which will be licensed: ${ }^{3}$

\section{(33) LF 1}

exh $\mathrm{K}[\mathrm{A}$ or $\mathrm{B}]$

$=K(A \vee B) \wedge \neg K(A) \wedge \neg K(B) \wedge \neg K(A \wedge B)$

\section{(34) LF 2}

$$
\begin{aligned}
& \operatorname{exh} \mathrm{K} \operatorname{exh}[\mathrm{A} \text { or } \mathrm{B}] \\
& =K(A \vee B) \wedge \neg K(A) \wedge \neg K(B) \wedge K \neg(A \wedge B)
\end{aligned}
$$

The only difference between these two LFs is the attitude entailed about the corresponding conjunction: while LF 1 entails an uncertainty implicature about [A and B], LF 2 entails the scalar implicature that the speaker is certain that [A and B] is false. Note that LF 3 violates Epistemic Transparency because it doesn't entail any attitude about the relevant alternatives:

\section{*LF 3}

$\mathrm{K} \operatorname{exh}[\mathrm{A}$ or $\mathrm{B}]$

$=K(A \vee B) \wedge K \neg(A \wedge B)$

$\mathcal{A} L T=\{\ldots, \mathrm{K} \operatorname{exh} \mathrm{A}, \mathrm{KA}, \mathrm{K} \operatorname{exh} \mathrm{B}, \mathrm{KB}, \ldots\}$

$\boldsymbol{x}$ Epistemic Transparency

With these basics in place we can check the predictions of the new theory for our original minimal pair, repeated below:

(36) a. Mary drank some of the beers. $=$ SOME

b. Mary drank some or all of the beers. $=$ SOME OR ALL

In parallel to the simple disjunction just reviewed, there are two ET-licensed LFs for the simple sentence SOME:

\section{LF 1}

exh $\mathrm{K}$ SOME

$=K(S O M E) \wedge \neg K(A L L)$

3 Note that both LFs entail ignorance about the disjuncts, i.e., $\neg K(A) \wedge \neg K(\neg A) \wedge \neg K(B) \wedge \neg K(\neg B)$. 
(38) LF 2

$\mathrm{K}$ exh SOME

$=K(S O M E) \wedge K \neg(A L L)$

Thus, just like a simple disjunction, the sentence SOME is predicted to either have an uncertainty implicature or a scalar implicature. In this the theory makes the same prediction as the Neo-Gricean pragmatic theory.

Now we can turn to the predictions for the complex structure in (36b). Recall first that Hurford's Constraint forces us to adjoin exh within the first disjunct. Importantly, among the structures that satisfy HC, Epistemic Transparency will rule out all but one:

\section{LF 1 \\ $\operatorname{exh}_{2} \mathrm{~K}\left[\left[\operatorname{exh}_{1} \mathrm{SOME}\right]\right.$ or ALL $]$}

We will compute the denotation of this LF step by step. First, I assume uncontroversally that the embedded exhaustivity operator $e x h_{1}$ adds the negation of ALL to the meaning of its prejacent; the denotation of the embedded proposition is thus:

$$
\begin{aligned}
& \llbracket \mathrm{K}\left[\left[\operatorname{exh}_{1} \mathrm{SOME}\right] \text { or } \mathrm{ALL}\right] \rrbracket= \\
& K((S O M E \wedge \neg A L L) \vee A L L)=K(S O M E)
\end{aligned}
$$

The next step is to check which alternatives $e x h_{2}$ can exclude. Given the meaning of $e x h_{2}$ 's prejacent, these are the following:
a. $\mathcal{A L T} T_{2}=\left\{\mathrm{K} \operatorname{exh}_{1}\right.$ SOME, K ALL, K SOME $\}$
b. $I E_{2}=\left\{\mathrm{K} e x h_{1}\right.$ SOME, K ALL $\}$

The denotation of (42) is thus the following:

$$
\begin{aligned}
& \text { LF } 1 \\
& \operatorname{exh}_{2} \mathrm{~K}\left[\left[e^{x} h_{1} \text { SOME }\right] \text { or ALL }\right] \\
& =K(S O M E) \wedge \neg K(S O M E \wedge \neg A L L) \wedge \neg K(A L L) \\
& =K(S O M E) \wedge \neg K(\neg A L L) \wedge \neg K(A L L)
\end{aligned}
$$


Deriving Hurford's Constraint

(43) Matrix K Theory: Simple vs. Complex Structures

a. Simple Structure: $W$

i. $\operatorname{exh} \mathrm{K} W$

$$
=K(W) \wedge \neg K(S)
$$

ii. $\mathrm{K} \operatorname{exh} W$

$$
=K(W) \wedge K(\neg S)
$$

b. Complex Structure: $W$ or $S$

i. $\operatorname{exh}_{2} \mathrm{~K}\left[\left[\operatorname{exh}_{1} W\right]\right.$ or $\left.S\right]$

$$
=K(W) \wedge \neg K(S) \wedge \neg K(\neg S)
$$

Thus, the theory differs from Fox's grammatical theory of scalar implicature in that only the complex, but not the simple structure is predicted to entail a true ignorance implicature about the stronger alternative $S$.

There is one last step missing in this argument, however. While the LF in (42) satisfies Epistemic Transparency, we have not shown yet that no other structure does. Given that we still assume Hurford's Constraint as part of the theory at this point, there are two other possible LFs for the complex sentence, given below. As we will see, both are ruled out by the principle of ET:

$$
\begin{aligned}
& \text { LF } 2 \\
& \mathrm{~K}\left[\left[\operatorname{exh}_{1} \mathrm{SOME}\right] \text { or ALL }\right] \\
& =K(S O M E)
\end{aligned}
$$

This LF does not entail an epistemic attitude about the alternative ALL and is therefore ruled out by ET. As the reader can check for herself, an exhaustivity operator just below $\mathrm{K}$ will be vacuous, so that the resulting LF will likewise violate ET. LF 1 is indeed the only predicted structure for the complex sentence - given that Hurford's Constraint is assumed to be valid.

In sum, here are the predictions of the grammatical and the Matrix $\mathrm{K}$ theory (since the Neo-Gricean theory faces an independent problem with complex structures, as reviewed above, I will limit the comparison to these two here.):

\section{Matrix K Theory}

a. $W$

Uncertainty Implicature $\neg \mathrm{K}(S)$, Scalar implicature $\mathrm{K}(S)$

b. $W$ or $S$

Ignorance Implicature $\neg \mathrm{K}(S) \wedge \neg \mathrm{K} \neg(S)$ 


\section{Grammatical cum pre-Neo-Gricean Theory}

a. $W$

Ignorance Implicature $\neg \mathrm{K}(S) \wedge \neg \mathrm{K} \neg(S)$, Scalar implicature $\mathrm{K} \neg(S)$

b. $W$ or $S$

Ignorance Implicature $\neg \mathrm{K}(S) \wedge \neg \mathrm{K} \neg(S)$

At this point it becomes important to empirically check these subtle differences. Consider the following contrasts:

a. Mary had a sandwich or a cookie.

b. $\checkmark$ Are you saying you don't know which she had?

The question targets the ignorance implicature about the disjuncts (e.g., $\neg$ KCOOKIE $\wedge \neg \mathrm{K} \neg$ (COOKIE), which are entailed by the semantic meaning in the Matrix K theory and a pragmatic implicature under the grammatical theory. Note that the grammatical theory predicts that a simple disjunction A OR B as in (47a) may also give rise to an ignorance implicature about the conjunction (see (46a) in (46a) above; this is because of the two symmetric alternatives A AND B and A AND B AND NOT BOTH). However this implicature behaves differently under the same conditions:

a. Mary had a sandwich or a cookie.

b. \# Are you saying you don't know whether she had both?

c. \# Are you saying she might have had both?

The Matrix K theory on the other hand doesn't predict the ignorance implicature in question to be entailed, as shown in (45). Thus, while the contrast between (47) and (48) is unexpected under the grammatical theory, it is predicted by the Matrix K theory. Second, consider now the behavior of complex structures:

(49) a. Mary had a sandwich or a cookie or both.

b. $\checkmark$ Are you saying you don't know whether she had both?

c. $\checkmark$ Are you saying she might have had both?

The contrast between the simple (48) and the complex (49) is again predicted by the Matrix K, but not the grammatical theory: the latter, but not the former structure entails ignorance about the conjunction. Though these facts deserve more discussion, I will confine myself to pointing them out here. 
Deriving Hurford's Constraint

\section{Deriving Hurford's constraint}

In the last section we saw that the Matrix K theory offers an empirical advantage over the two standard theories of implicature. In this section we will see that the theory also allows us to get rid of Hurford's constraint. As the constraint is stipulative, this is an important improvement.

Intuitively, Hurford's constraint should fall out as a corollary of Grice's Brevity. This is because disjunctions in which one disjunct entails the other are semantically equivalent to the weaker disjunct (example from Fox \& Spector (2009/2013)):

(50) \#Mary owns a dog or a German Shepherd.

$\equiv$ Mary owns a dog.

Given this equivalence, the second disjunct is adding syntactic complexity without any semantic effect. I propose the following principle to formalize this - again using Katzir's notion of structural complexity:

\section{(51) Efficiency}

An LF $\phi$ is ruled out if there is a distinct competitor $\psi$ such that

a. $\psi \in \mathcal{A L T}(\phi)$

b. $\llbracket \psi \rrbracket=\llbracket \phi \rrbracket$

Consider first what Efficiency would predict for the complex structures W OR S we have discussed above. In a theory without Matrix $\mathrm{K}$, the constraint would rule out these structures even under the parse with local exh (i.e., the LF which Hurford's constraint would necessitate, containing an embedded exh in the weaker disjunct). Generally, the felicity of these complex structures will be problematic for any formalization of Brevity in terms of equivalence with a sub-constituent, as the structure below illustrates:

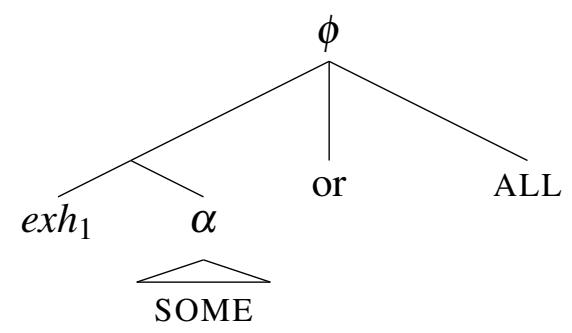

Since $\llbracket \phi \rrbracket=\llbracket \alpha \rrbracket$ and $\alpha \in \mathcal{A} L T(\phi),(52)$ would be ruled out just like the actually infelicitous (50).

Let us now check how Efficiency works within the theory proposed here. To anticipate the result, all but the following LFs for the complex W OR S will be ruled out (illustrated here for SOME OR ALL but as before the results carry over to A OR B OR BOTH): 


$$
\begin{aligned}
& \left.e x h_{2} \mathrm{~K}\left[\left[e x h_{1} \mathrm{SOME}\right] \text { or } \mathrm{ALL}\right]\right] \\
& =K(\mathrm{SOME}) \wedge \neg K(\mathrm{ALL}) \wedge \neg K \neg(\mathrm{ALL})
\end{aligned}
$$

The structural alternatives against which this LF competes are shown below, together with their denotations:

$$
\begin{aligned}
& \mathcal{A L T}=\left\{\mathrm{K} \operatorname{exh}_{1} W, \mathrm{~K} S, \operatorname{exh}_{2} \mathrm{~K} W, \ldots\right\} \\
& =\{[K(W \wedge K(\neg S)], K(S),[K(W) \wedge \neg K(S)], \ldots\}
\end{aligned}
$$

Since no alternative LF is equivalent to (52), the structure therefore satisfies Efficiency. What about other possible LFs for [W OR S]? Recall that admissible structures also have to satisfy the principle of Epistemic Transparency. This leaves the following LFs:

$$
\begin{aligned}
& \operatorname{exh} \mathrm{K}[\text { SOME OR ALL] } \\
& =K(S O M E) \wedge \neg K(A L L)
\end{aligned}
$$

This LF is empirically inadequate, but it does satisfy ET: the alternatives about which an epistemic attitude has to be entailed are just the singleton set $\{$ ALL , and the LF entails that the speaker is not certain about this alternative. Consider Efficiency, however. The competing LFs are given below:

$$
\begin{aligned}
& \mathcal{A} L T=\{\text { exh } \mathrm{K} \text { some }, \ldots\} \\
& =\{K(S O M E) \wedge \neg K(A L L)\}
\end{aligned}
$$

The meaning of this simpler alternative is equivalent to (55), which ruled out this structure. There is only one other structure which would be licensed by Epistemic Transparency:

$$
\begin{aligned}
& \mathrm{K} \operatorname{exh}[\mathrm{SOME} \text { OR ALL] } \\
& =K(S O M E) \wedge K(\neg A L L)
\end{aligned}
$$

However, Efficiency correctly rules out this structure, as it is equivalent to a simpler alternative LF:

$$
\begin{aligned}
& \mathcal{A} L T=\{\mathrm{K} \text { exh SOME }\} \\
& =\{K(S O M E) \wedge K(\neg A L L)\}
\end{aligned}
$$

In sum, together with Epistemic Transparency, the principle of Efficiency allows us to get rid of Hurford's constraint. Only the LF in (53) is predicted to be licensed: the ignorance implicatures it entails make it non-equivalent to any simpler LF.

In this section we offered a formalization of Grice's Brevity maxim. In the Matrix $\mathrm{K}$ theory, the proposed principle of Efficiency predicts that complex structures $\mathrm{W}$ OR $S$ are licensed only under a reading which entail ignorance about $S$. In the 
Deriving Hurford's Constraint

previous section we saw that there is empirical evidence which corroborates this. What remains to be done is to verify if the theory as defined so far can be extended to complex structures like (50) which are infelicitous. This will be the topic of the next section.

\section{Extending the theory to infelicitous complexity}

So far we have discussed complex structures of the form W OR S which are felicitous. Under the theory proposed here, they are licensed because they are efficient in expressing a meaning which no simpler structure could express. Their distinctive feature are ignorance implicatures. However, some complex structures in which one disjunct entails the other are in fact infelicitous (repeated from (50)):

(59) \# Mary owns a dog or a German Shepherd.

The infelicitous cases differ from the felicitous ones in that the entailment relation between the (surface) disjunct is contextual: that Fido is a German Shephard entails Fido is a dog is not a logical fact. I will use the following notation to indicate contextual or world-knowledge based entailment:

(60) Fido is a German Shepherd $\vDash_{W K}$ Fido is a dog

Though there isn't a logical relation between the two propositions, asserting a weaker (or, less specific) sentence can give rise to an inference about the corresponding stronger (or, more specific) sentence. Here I hypothesize that this inference can be modeled as an implicature (Grice 1975, Matsumoto 1995, Singh 2008):

(61) Where is Mary?

She is in France

$\leadsto \neg \mathrm{K}($ She is in $l), l$ a city in France

This uncertainty implicature arises in contexts in which city-level specificity is at stake, i.e., in contexts in which Mary's current city is the desired information. The observation is quite general. Using a specificity level below $n$ in contexts in which $n$-level specificity is required can result in uncertainty implicatures, but importantly, never in scalar implicatures:

(62) Where is Mary?

She is in France

$\rightsquigarrow \mathrm{K} \neg($ She is in $l), l$ a city in France

To derive this, I follow Singh (2008) in making the following assumption: 
(63) Specificity Alternatives

$\mathcal{A} L T($ FRANCE $)=\{$ Paris, Marseille, Lyon, ... $\}$

How does this account for the absence of scalar implicatures with specificity-based alternatives? Assume the alternatives of (61) could be given by the singleton set \{Mary is in Paris\}. Exhaustifying below $\mathrm{K}$ would then predict the missing scalar implicature:

(64) $\mathrm{K} \operatorname{exh}$ [Mary is in France]

$=K$ (mary is in france) $\wedge K \neg$ (mary is in paris)

Take on the other hand the full set of alternatives including all cities in France (assume that every location in France is part of a city). Exhaustification below $\mathrm{K}$ yields the following result now:

$\mathrm{K}$ exh [Mary is in France]

$=K$ (mary is in france $) \wedge K \neg($ mary is in paris $) \wedge K \neg($ mary is in marseille $) \wedge$ $\ldots \vDash \perp$

Before considering what this semantic denotation amounts to, there is a crucial assumption which we need to make explicit first. To be able to derive (65) at all, exhaustification needs to be blind to world-knowledge, e.g., facts about city locations or German Shepherd genetics - otherwise, the alternatives would not be innocently excludable. This is an assumption defended in Fox \& Hackl (2006); Singh (2008); Magri (2009) on the basis of independent considerations: ${ }^{4}$

\section{(66) Blindness}

Innocent Exclusion avoids logical but not contextual inconsistency

Having made explicit that and how (67) comes about, let us now check what the consequences are. Exhaustification below $\mathrm{K}$ will be possible in the grammar, but the meaning computed semantically is problematic when interpreted in context: it ascribes to the speaker the belief that Mary is in France but not at any location in France. This I argue will ultimately lead the hearer to re-parse the sentence as below:

$$
\begin{aligned}
& \text { exh } \mathrm{K} \text { [Mary is in France }] \\
& =K \text { (mary is in france }) \wedge \neg K \text { (mary is in paris }) \wedge \neg K(\text { mary is in marseille }) \wedge \\
& \ldots
\end{aligned}
$$

4 It should be noted that the infelicity of Hurford disjunctions like (59) can be derived in the present system even without Blindness. The reason is that, though possible LFs will no longer give rise to inconsistent implicatures, they will all violate the principle of Efficiency. 
This accounts for the observed uncertainty implicature in contexts like (61) above, as well as for the systematic absence of the scalar implicatures as illustrated in (62).

Let us now return to the main concern of this section, namely, complex sentences in which on disjunct contextually entails the other. Adding Blindness in (66), and the assumption about Specificity scales in (63), any possible LF for these sentences will either be inconsistent in the way (67) is, or ruled out by the principles of Epistemic Transparency and/or Efficiency.

To show this, we can start with a very simple LF:

\section{(68) LF 1}

$$
\begin{aligned}
& \mathrm{K} \text { [Mary is in France or in Paris] } \\
& =K(\text { FRANCE })
\end{aligned}
$$

As before I will use an abbreviation, FRANCE OR PARIS, in what follows. The LF above violates both of the two central principles. It doesn't entail an attitude about the alternatives \{PARIS, MARSEILle, ... \}, as can easily be read off (68). Secondly, it is also equivalent to its simpler alternative KFRANCE, thereby violating Efficiency.

Let us look at structures which satisfy ET now.

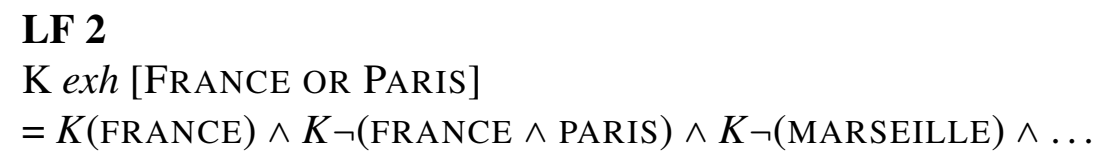

LF 2

$\mathrm{K} \operatorname{exh}$ [FRANCE OR PARIS]$$
=K(\text { FRANCE }) \wedge K \neg(\text { FRANCE } \wedge \text { PARIS }) \wedge K \neg(\text { MARSEILLE }) \wedge \ldots
$$

Note that FRANCE $\wedge$ PARIS is contextually equivalent to PARIS. (69) is therefore contextually equivalent to (65), repeated below. As this is a simpler alternative, (69) will be ruled out by Efficiency, in addition to being inconsistent when interpreted relative to world knowledge:

$$
\begin{aligned}
& \mathcal{A} L T((69))=\{\mathrm{K} \text { exh FRANCE }, \ldots\} \\
& =K(\text { FRANCE }) \wedge K \neg(\text { PARIS }) \wedge K \neg(\text { MARSEILLE }) \ldots \\
& \mathbf{X} \text { Efficiency }
\end{aligned}
$$

What happens if exh is adjoined above K? This will avoid the contextual contradiction of (70), viz., that the speaker is certain that Mary is in France and also certain that she is not at $l$, for all locations in France. But the corresponding LF is ruled out by Efficiency:

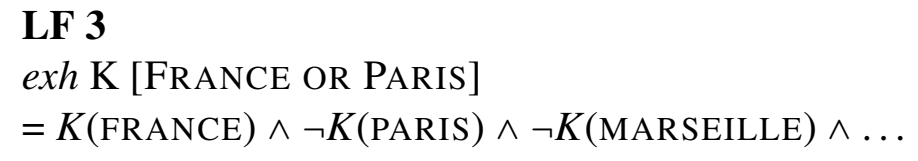

LF 3 exh K [FRANCE OR PARIS]$$
=K(\text { FRANCE }) \wedge \neg K(\text { PARIS }) \wedge \neg K(\text { MARSEILLE }) \wedge \ldots
$$

This semantic denotation will not be inconsistent when interpreted in context: a speaker may actually be certain that Mary is in France without being certain about any more specific place. The only problem with (71) is that this meaning could be expressed more efficiently with one of its competitor LFs, shown below: 


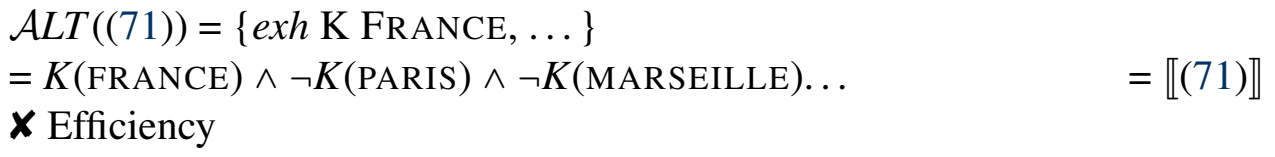

There is one more LF which should be checked, namely, the structure which in SOME OR ALL was predicted to be licensed. It is given below:

\section{LF 4 $e x h_{2} \mathrm{~K}\left[\left[\operatorname{exh}_{1}\right.\right.$ FRANCE] OR PARIS]}

To compute the meaning of this LF, the first step is to compute the meaning of the prejacent of $e x h_{2} ; \llbracket e x h$ FRANCE $\rrbracket$ was already given in (70) above:

$$
\begin{aligned}
& \llbracket \mathrm{K}\left[\left[\operatorname{exh}_{1} \text { FRANCE }\right] \text { OR PARIS }\right] \rrbracket= \\
& K[(\text { FRANCE } \wedge \neg \text { PARIS } \wedge \neg \text { MARSEILLE. . }) \vee \text { PARIS }]
\end{aligned}
$$

What are the alternatives which $e x h_{2}$ in (76) quantifies over and which of them are innocently excludable? Recall that exh is blind to the contextual relations between the alternatives. Therefore, it cannot see that the denotation in (74) is contextually equivalent to KPARIS (this is because the first disjunct in (74) is a contextual contradiction). Therefore, one logically innocently excludable alternative of $e x h_{2}$ will be the following:

$$
\begin{aligned}
& \left.\left.\mathcal{A} L T_{2}=\left\{\operatorname{Kexh}_{1} \text { FRANCE, KPARIS, K[[exh } h_{1} \text { FRANCE }\right] \text { AND PARIS }\right]\right\} \\
& I E_{2}=\{\text { KPARIS }, \ldots\}
\end{aligned}
$$

The meaning of LF 4 will therefore be:

$$
\begin{aligned}
& \text { LF } 4 \\
& e x h_{2} \text { K }\left[\left[e x h_{1} \text { FRANCE }\right] \text { OR PARIS }\right] \\
& =K[(\text { FRANCE } \wedge \neg \text { PARIS } \wedge \neg \text { MARSEILLE... }) \vee \text { PARIS }] \wedge \neg \text { KPARIS }
\end{aligned}
$$

Semantic computation is now completed and we can factor in world knowledge. Specifically:

$$
\begin{aligned}
& K[(\text { FRANCE } \wedge \neg \text { PARIS } \wedge \neg \text { MARSEILLE } \ldots) \vee \text { PARIS }] \wedge \neg K \text { PARIS } \\
& \vDash_{W K} K \text { PARIS } \wedge \neg \text { PPARIS }
\end{aligned}
$$

It is easy to see that this is inconsistent. In sum, then, although LF 4 seems to satisfy Efficiency and Epistemic Transparency (the only LF to do so), when interpreted relative to world knowledge it will yield a contradiction.

In sum, we saw that the theory can be extended to infelicitous complex structures of the form W OR S under two independently motivated assumptions (see (63) and (66)), which can also account for the specific behavior of specificity-based inferences 
Deriving Hurford's Constraint

(see Singh 2008). The predictions that emerge are empirically correct: disjunctions like DOG OR GERMAN SHEPHERD are infelicitous. In our proposal, this is because any available structure is either contextually inconsistent or violates the principles of Efficiency and ET. Thus, we can indeed get rid of Hurford's constraint, as suggested in the last section.

\section{Conclusion and outlook}

This paper offers a new theory of grammatical implicatures and their interaction with pragmatics. In this new theory, it is possible to derive Hurford's constraint from a principle of economy, which I have called Efficiency here. There are several lines of future research that emerge from this paper. First, the empirical scope of the principle of Efficiency, and its relation to other cases of seeming redundancy and to (embedded) exhaustification. Second, a thorough re-examination of the scope of (Gricean) pragmatics and specifically, its relation to Epistemic Transparency. Third, a finer-grained characterization of the Matrix K operator, locating it within broader questions about the semantics and pragmatics of evidential and epistemic operators. Lastly, a closer look at specificity-based implicatures, including the status of the Blindness hypothesis, in deriving the apparent absence of scalar implicatures and presence of uncertainty implicatures.

\section{References}

Alonso-Ovalle, Luis. 2006. Disjunction in Alternative Semantics: UMass Amherst $\mathrm{PhD}$ dissertation.

Chierchia, Gennaro. 2006. Broaden your views: Implicatures of domain widening and the "logicality" of language. Linguistic Inquiry 37(4). 535-590.

Fox, Danny. 2007. Free choice and the theory of scalar implicatures. In Uli Sauerland \& Penka Stateva (eds.), Presupposition and Implicature in Compositional Semantics, 71-120. New York: Palgrave Macmillan.

Fox, Danny \& Martin Hackl. 2006. The universal density of measurement. Linguistics and Philosophy 29(5). 537-586.

Fox, Danny \& Benjamin Spector. 2009/2013. Economy and embedded exhaustification. Handout/ms., MIT and ENS.

Hurford, James. 1974. Exclusive or inclusive disjunction. Foundations of Language 11. 409-411.

Katzir, Roni. 2007. Structurally-defined alternatives. Linguistics and Philosophy 30(6). 669-690.

Magri, Giorgio. 2009. A theory of individual-level predicates based on blind mandatory scalar implicatures. Natural Language Semantics 17(3). 245-297. 
Meyer, Marie-Christine. 2013. Ignorance and Grammar: MIT PhD dissertation. Sauerland, Uli. 2004. Scalar implicatures in complex sentences. Linguistics and Philosophy 27(3). 367-391.

Singh, Raj. 2008. On the interpretation of disjunction: Asymmetric, incremental, eager for inconsistency. Linguistics and Philosophy 31(2). 245-260.

\author{
Marie-Christine Meyer \\ Language, Logic and Cognition Center \\ The Hebrew University of Jerusalem \\ Mount Scopus \\ 91905 Jerusalem \\ Israel \\ macrst@alum.mit.edu
}

